\title{
Consenso de Sistemas Multiagentes por Realimentação Dinâmica de Saída via LMIs *
}

\author{
Bruno M. C. Silva* João Y. Ishihara * Eduardo S. Tognetti * \\ * Departamento de Engenharia Elétrica, Universidade de Brasília, DF, \\ (e-mail: brunocalazans.s@hotmail.com, ishihara@ene.unb.br, \\ estognetti@ene.unb.br).
}

\begin{abstract}
:
This work proposes the design of protocol for consensus of linear multiagent systems by reducedorder dynamic output feedback. All the agents have identical linear dynamics and only the output information of each agents is delivered throughout the communication network. Sufficient conditions are proposed for state consensus by means of Linear Matrix Inequalities (LMIs). Unlike the literature, rank constraints on the input and output matrices of the agents are not required. The effectiveness of the tuned protocol is illustrated by numerical experiment.

Resumo: Este trabalho propõe o projeto de protocolo para consenso de sistemas multiagentes lineares por realimentação dinâmica de saída de ordem reduzida. Todos os agentes possuem dinâmicas lineares idênticas e apenas a informação de saída de cada agente é entregue por toda a rede de comunicação. São propostas condições suficientes para consenso de estado por meio de Desigualdades Matriciais Lineares (em inglês, Linear Matrix Inequalities - LMIs). Diferente da literatura, restrições de posto sobre as matrizes de entrada e saída dos agentes não são requeridas. A efetividade do protocolo sintonizado é ilustrada por experimento numérico.
\end{abstract}

Keywords: Multi-agent Systems, Linear Systems, Consensus, Linear Matrix Inequalities, Dynamical Output Feedback.

Palavras-chaves: Sistemas Multiagentes, Sistemas Lineares, Consenso, Desigualdades Matriciais Lineares, Realimentação Dinâmica de Saída.

\section{INTRODUÇÃO}

Sistemas Multiagentes (SMA) têm recebido grande atenção em diversas áreas, devido ao seu imenso potencial de aplicação como em controle cooperativo de veículos aéreos não tripulados (VANTs) (Kolaric et al., 2018), controle de múltiplos satélites (Zhang and Gurfil, 2018) e manipuladores robóticos (Ren et al., 2018), assim como computação na nuvem, mídias sociais, construção civil, entre outras (Dorri et al., 2018).

A principal característica dos sistemas multiagentes é a implementação de vários nós distribuídos ao redor de uma planta, cada um conectado com seu vizinho através de uma rede de comunicação. Esse esquema torna possível o controle cooperativo de vários subsistemas, havendo troca de informação, que é realizada para alcançar um propósito desejado. Assim, para que haja cooperação entre os subsistemas, é necessário um algoritmo ou protocolo, que força cada agente a obter consenso com seus vizinhos, criando um comum acordo em relação a uma variável do problema (Olfati-Saber et al., 2007).

Uma das abordagens do problema de consenso de SMA encontrada na literatura é a utilização de protocolos com Realimentação Dinâmica de Saída (RDS), como por exemplo Liu and Jia (2010) e Gao et al. (2014), que resolvem o problema $H_{\infty}$ para os casos contínuo e discreto, respec-

^ Apoiado pelo CNPQ (312528/2017-5), CAPES e FAPDF. tivamente, considerando que a rede de comunicação dos agentes sofre mudanças de topologia. Nesses trabalhos, as topologias de comunicação dos agentes são modeladas por grafos não direcionados. Ambos trabalhos consideram que a matriz da entrada do sistema possui posto coluna pleno.

Trabalhos mais recentes na literatura solucionam o problema de consenso de SMA por RDS envolvendo situações de maior complexidade, como em Min et al. (2018) que considera agentes com estados ruidosos, além de um controlador dinâmico de saída que possui saltos Markovianos em seus parâmetros. Em Ao et al. (2018) é investigado o problema $H_{\infty}$, considerando que as entradas dos agentes possuem atrasos variantes no tempo. Assim, como nos trabalhos citados anteriormente, as soluções destes problemas são baseadas em projetos que assumem posto coluna pleno para a matriz de entrada dos agentes.

Uma solução do problema de RDS para SMA, sem restrições nos parâmetros dos agentes, é apresentada por Li et al. (2018). Neste trabalho são considerados agentes incertos e dois tipos de controladores dinâmicos com uma forma particular, sendo uma das principais contribuições desse trabalho, a utilização de uma rede direcionada generalizada.

Uma outra forma encontrada na literatura de solucionar o problema de RDS para SMA, sem restringir a estrutura dos parâmetros do sistema, é apresentada em Zhao and Park (2015), que obtém uma desigualdade matricial bili- 
near (BMI), posteriormente tratada como desigualdades matriciais lineares (LMIs), através do método da homotopia (Richter and DeCarlo, 1984). Nessa abordagem fixa-se a matriz de Lyapunov ou os parâmetros do controlador dinâmico, e são resolvidas LMIs através de um método iterativo.

Como foi visto, esse tipo de problema apresenta uma dificuldade, que é a obtenção de uma solução que não imponha restrições para as matrizes de entrada e saída dos agentes. Considerando uma rede homogênea, onde todos os agentes possuem a mesma lei dinâmica, será apresentada uma solução para o problema de consenso baseado em um protocolo de consenso dinâmico de saída, onde os parâmetros dos controladores dinâmicos de ordem reduzida são obtidos através da solução de LMIs. A seguir são apresentadas as principais contribuições do presente artigo:

- A solução para o problema de consenso de SMA por RDS, com o uso de um protocolo de consenso que torna possível um projeto sem restrições nas matrizes do SMA.

- A técnica apresentada por este trabalho oferece uma solução através de um problema convexo para o problema proposto, diferente de Zhao and Park (2015), que apresenta uma solução através de um método iterativo para encontrar a solução de uma BMI.

Notação: Uma matriz $Y$ definida positiva (negativa) é denotada por $Y>0(Y<0)$. Para uma matriz qualquer $Y$, denota-se por $Y^{\perp}$ qualquer matriz cujas colunas formam uma base para o espaço nulo de $Y$. O símbolo * representa um termo simétrico em uma matriz definida em termos de blocos. A notação $\mathbf{1}_{m}$ diz respeito ao vetor coluna preenchido com $m$ elementos 1 . A notação $H e\{\}$ representa a soma de uma matriz por sua transposta. A operação $\otimes$ é chamada de produto de Kronecker.

\section{PRELIMINARES}

\subsection{Teoria de Grafos}

Um grafo direcionado é denotado por $\mathcal{G}(\mathcal{V}, \mathcal{E}, \mathcal{A})$, onde $\mathcal{V}=\left\{v_{1}, \ldots, v_{m}\right\}$ é o conjunto de vértices e $\mathcal{E}=\left\{e_{j i}\right\}$ é o conjunto de arestas conectadas a cada vértice. As arestas são definidas por $e_{j i}=\left(v_{j}, v_{i}\right)$, onde o primeiro elemento $v_{j}$ é o vértice pai (ou nó pai) e o elemento $v_{i}$ é o vértice filho (ou nó filho). A matriz de adjacência associada ao grafo $\mathcal{G}$ é definida por $\mathcal{A}=\left[a_{i j}\right]$, com $a_{i j}>0$ se $\exists e_{j i}$, e $a_{i j}=0$ caso contrário.

A matriz do grau dos vértices $\mathcal{D}=\left[d_{i i}\right]$ é uma matriz diagonal com elementos $d_{i i}=\sum_{j=1}^{m} a_{i j}$. A partir dessa definição, tem-se que a matriz Laplaciana pode ser calculada por

$$
L=\mathcal{D}-\mathcal{A}
$$

ou, $L=\left[l_{i, j}\right]$ com $l_{i i}=\sum_{j=1}^{m} a_{i j}$ e $l_{i j}=-a_{i j}$, para $i \neq j$. Sendo $L 1_{m}=0_{m}$ uma importante propriedade da matriz Laplaciana (Godsil and Royle, 2001).

Neste trabalho será considerado um único grafo que modela a rede de comunicação entre os agentes, i.e., a topologia de comunicação entre eles será fixa.

Seguindo Li et al. (2018), será assumida a hipótese a seguir.
Hipótese 1. O grafo $\mathcal{G}$ contém uma árvore de extensão direcionada.

A Hipótese 1 é conhecida como pré-requisito fundamental para a solução do problema de consenso, garantindo que haja uma rede de comunicação que conecta todos os agentes por pelo menos um caminho direcionado.

\subsection{O Problema de Consenso de Sistemas Multiagentes} por Realimentação Dinâmica de Saída com Topologia Fixa

Considere $m$ agentes em uma rede direcionada, descritos pelo sistema a seguir

$$
\begin{aligned}
\dot{x}_{i}(t) & =A x_{i}(t)+B u_{i}(t), \quad i=1, \ldots, m \\
y_{i}(t) & =C x_{i}(t),
\end{aligned}
$$

onde $x_{i}(t) \in \mathbb{R}^{n}$ são as variáveis de estado, $y_{i}(t) \in \mathbb{R}^{q}$ as saídas, $u_{i}(t) \in \mathbb{R}^{s}$ as entradas de controle, $A \in \mathbb{R}^{n \times n}$ a matriz de transmissão de estados, $B \in \mathbb{R}^{n \times s}$ matriz de entrada e $C \in \mathbb{R}^{q \times n}$ a matriz de saída. Cada agente é controlado localmente por cada um dos $m$ controladores dinâmicos a seguir

$$
\begin{aligned}
\dot{x}_{c, i}(t) & =A_{c} x_{c, i}(t)+B_{c} \nu_{i}(t), \quad i=1, \ldots, m \\
u_{i}(t) & =C_{c} x_{c, i}(t)+D_{c} \nu_{i}(t),
\end{aligned}
$$

onde $x_{c, i}(t) \in \mathbb{R}^{n_{c}}$ são as variáveis de estado dos controladores dinâmicos, $A_{c} \in \mathbb{R}^{n_{c} \times n_{c}}, B_{c} \in \mathbb{R}^{n_{c} \times n_{\nu}}, C_{c} \in \mathbb{R}^{s \times n_{c}}$, $D_{c} \in \mathbb{R}^{s \times n_{\nu}}$ os parâmetros dos controladores dinâmicos a serem encontrados e, $\nu_{i}(t) \in \mathbb{R}^{n_{\nu}}$ o protocolo de consenso, dado por

$$
\nu_{i}(t)=-\sum_{j \neq i, j=1}^{m} a_{i j} K\left(y_{i}(t)-y_{j}(t)\right), \quad i=1, \ldots, m .
$$

O sinal gerado pelo protocolo $\nu_{i}(t)$ é obtido pela realimentação das saídas dos agentes vizinhos ao agente $i$, conforme a rede de comunicação definida pelo grafo $\mathcal{G}$. A matriz $K \in \mathbb{R}^{n_{\nu} \times q}$ é o ganho a ser sintonizado. Neste trabalho é considerado que os controladores dinâmicos de saída (3) possuem ordem reduzida, i.e., $n_{c}<n$.

Definição 2. Dizemos que o sistema multiagente com realimentação dinâmica de saída (2)-(4) atinge consenso, se e somente se, para todo $i \neq j, i, j=1, \ldots, m$, os estados dos agentes satisfazem

$$
\lim _{t \rightarrow \infty}\left(x_{i}(t)-x_{j}(t)\right)=0 .
$$

\subsection{Sistema Multiagente Transformado}

O sistema aumentado, considerando os $m$ agentes em (2) é dado por

$$
\begin{aligned}
& \dot{x}(t)=\left(I_{m} \otimes A\right) x(t)+\left(I_{m} \otimes B\right) u(t), \\
& y(t)=\left(I_{m} \otimes C\right) x(t),
\end{aligned}
$$

em que,

$$
\begin{aligned}
& x(t)=\left[\begin{array}{llll}
x_{1}(t)^{T} & x_{2}(t)^{T} & \cdots & x_{m}(t)^{T}
\end{array}\right]^{T}, \\
& y(t)=\left[\begin{array}{llll}
y_{1}(t)^{T} & y_{2}(t)^{T} & \cdots & y_{m}(t)^{T}
\end{array}\right]^{T}, \\
& u(t)=\left[\begin{array}{llll}
u_{1}(t)^{T} & u_{2}(t)^{T} & \cdots & u_{m}(t)^{T}
\end{array}\right]^{T} .
\end{aligned}
$$

Da mesma forma, o controlador dinâmico aumentado é dado por 


$$
\begin{aligned}
\dot{x}_{c}(t) & =\left(I_{m} \otimes A_{c}\right) x_{c}(t)+\left(I_{m} \otimes B_{c}\right) \sum_{k=1}^{m} \bar{\nu}_{k}(t), \\
u(t) & =\left(I_{m} \otimes C_{c}\right) x_{c}(t)+\left(I_{m} \otimes D_{c}\right) \sum_{k=1}^{m} \bar{\nu}_{k}(t),
\end{aligned}
$$

onde o protocolo de consenso de saída e a variável de estado do controlador dinâmico, respectivamente, são escritos como

$$
\begin{aligned}
\bar{\nu}_{k}(t) & =-\left(L_{k} \otimes K C\right) x(t), \\
x_{c}(t) & =\left[\begin{array}{llll}
x_{c, 1}(t)^{T} & x_{c, 2}(t)^{T} \cdots x_{c, m}(t)^{T}
\end{array}\right]^{T},
\end{aligned}
$$

sendo $\bar{\nu}_{k}(t)$ o vetor de entrada do $k$-ésimo controlador dinâmico, na forma $\varrho_{k} \otimes u_{k}(t)$, com $\varrho_{k} \in \mathbb{R}^{n m}$ possuindo a $k$-ésima entrada sendo um e as outras sendo zero, e $L_{k}$ é a $k$-ésima matriz Laplaciana do subgrafo considerando somente as arestas que apontam para o vértice $v_{k}$.

Substituindo o controlador dinâmico aumentado (10) no sistema (6), é obtido o seguinte sistema em malha fechada

$$
\begin{aligned}
\underbrace{\left[\begin{array}{c}
\dot{x}(t) \\
\dot{x}_{c}(t)
\end{array}\right]}_{\dot{\bar{x}}(t)} & =\underbrace{\left[\begin{array}{cc}
\left(I_{m} \otimes A\right) & \left(I_{m} \otimes B C_{c}\right) \\
0 & \left(I_{m} \otimes A_{c}\right)
\end{array}\right]}_{\mathrm{A}} \underbrace{\left[\begin{array}{c}
x(t) \\
x_{c}(t)
\end{array}\right]}_{\bar{x}(t)} \\
& -\sum_{k=1}^{m} \underbrace{\left[\begin{array}{cc}
L_{k} \otimes\left(B D_{c} K C\right) & 0 \\
L_{k} \otimes\left(B_{c} K C\right) & 0
\end{array}\right]}_{\underline{\mathrm{B}}_{k}} \underbrace{\left[\begin{array}{c}
x(t) \\
x_{c}(t)
\end{array}\right]}_{\bar{x}(t)} .
\end{aligned}
$$

De acordo com Sun and Wang (2009), uma transformação do tipo de árvore pode ser aplicada ao sistema (11), para reescrever o problema de consenso como um problema de estabilidade, introduzindo uma nova variável que representa o desacordo entre os agentes. Essa transformação é importante, pois reduz o número de variáveis de (6) e (10), de $m$ para $m-1$, o que reduz a ordem do sistema (11), e além disso, transforma a matriz laplaciana do grafo, em uma matriz de dimensão menor.

O problema de consenso segundo a Definição 2, pode ser transformado em um problema de estabilidade, através de uma mudança de variável, que considera o desacordo dos estados dos agentes $z_{1, i}=x_{1}(t)-x_{i+1}(t)$, assim como o desacordo dos estados do controlador dinâmico $z_{2, i}=x_{c, 1}(t)-x_{c, i+1}(t)$, tal que $i=1, \ldots, m-1$, também descrita por

$$
z(t)=\left[\begin{array}{l}
z_{1} \\
z_{2}
\end{array}\right]=\bar{U} \bar{x}(t)
$$

onde

$$
\begin{aligned}
\bar{U} & =\left[\begin{array}{cc}
U \otimes I_{n} & Z \otimes S_{n \times n_{c}} \\
Z \otimes S_{n_{c} \times n} & U \otimes I_{n_{c}}
\end{array}\right], \\
U & =\left[\begin{array}{ll}
\mathbf{1}_{m-1} & -I_{m-1}
\end{array}\right], \\
Z & =\left[\begin{array}{ll}
0_{m-1} & 0_{(m-1) \times(m-1)}
\end{array}\right],
\end{aligned}
$$

e $S_{n \times n_{c}}$ uma matriz qualquer. O estado $\bar{x}(t)$ pode ser recuperado pela expressão

$$
\bar{x}(t)=\left[\begin{array}{c}
\mathbf{1}_{m} \otimes x_{1}(t) \\
\mathbf{1}_{m} \otimes x_{c, 1}(t)
\end{array}\right]+\bar{W} z(t),
$$

sendo

$$
\begin{aligned}
& \bar{W}=\left[\begin{array}{cc}
W \otimes I_{n} & Z^{T} \otimes S_{n \times n_{c}} \\
Z^{T} \otimes S_{n_{c} \times n} & W \otimes I_{n_{c}}
\end{array}\right], \\
& W=\left[\begin{array}{c}
0_{m-1}^{T} \\
-I_{m-1}
\end{array}\right] .
\end{aligned}
$$

Então, derivada temporal de (12), fornece o sistema a seguir

$$
\dot{z}(t)=\bar{U}\left(\underline{\mathrm{A}} \bar{x}(t)-\sum_{k=1}^{m} \underline{\mathrm{B}}_{k} \bar{x}(t)\right) .
$$

Substituindo $\underline{\mathrm{A}}, \underline{\mathrm{B}}_{k}$ e (16) em (19), tem-se que

$$
\begin{aligned}
\dot{z}(t) & =\bar{U}\left[\begin{array}{c}
\left(\mathbf{1}_{m} \otimes A x_{1}(t)\right)+\left(\mathbf{1}_{m} \otimes B C_{c} x_{c, 1}(t)\right) \\
\left(\mathbf{1}_{m} \otimes A_{c} x_{c, 1}(t)\right)
\end{array}\right] \\
& +\bar{U}\left[\begin{array}{cc}
\left(I_{m} \otimes A\right) & \left(I_{m} \otimes B C_{c}\right) \\
0 & \left(I_{m} \otimes A_{c}\right)
\end{array}\right] \bar{W} z(t) \\
& -\sum_{k=1}^{m} \bar{U}\left[\begin{array}{c}
L_{k} \mathbf{1}_{m} \otimes\left(B D_{c} K C\right) x_{1}(t) \\
L_{k} \mathbf{1}_{m} \otimes x_{1}(t)
\end{array}\right] \\
& +\sum_{k=1}^{m} \bar{U}\left[\begin{array}{c}
L_{k} \otimes\left(B D_{c} K C\right) \\
L_{k} \otimes\left(B_{c} K C\right)
\end{array}\right] \bar{W} z(t) .
\end{aligned}
$$

Usando a propriedade $L_{k} \mathbf{1}_{m}=0_{m}$ e substituindo (13) e (17) em (20), é obtida a seguinte expressão

$$
\begin{aligned}
\dot{z}(t) & =\left[\begin{array}{c}
\left(U \mathbf{1}_{m} \otimes A x_{1}(t)\right)+\left(U \mathbf{1}_{m} \otimes B C_{c} x_{c, 1}(t)\right) \\
\left(U \mathbf{1}_{m} \otimes A_{c} x_{c, 1}(t)\right)
\end{array}\right] \\
& +\left[\begin{array}{cc}
(U W \otimes A) & \left(U W \otimes B C_{c}\right) \\
0 & \left(U W \otimes A_{c}\right)
\end{array}\right] z(t) \\
& -\sum_{k=1}^{m}\left[\begin{array}{cc}
U L_{k} W \otimes\left(B D_{c} K C\right) & 0 \\
U L_{k} W \otimes\left(B_{c} K C\right) & 0
\end{array}\right] z(t) .
\end{aligned}
$$

Observe que $U W=I_{m-1}$ e $U 1_{m}=0_{m}$. Assim, (21) pode ser reescrita da seguinte forma

$$
\dot{z}(t)=(\tilde{A}-\tilde{B}) z(t),
$$

onde,

$$
\begin{aligned}
& \tilde{A}=\left[\begin{array}{cc}
\left(I_{m-1} \otimes A\right) & \left(I_{m-1} \otimes B C_{c}\right) \\
0(m-1) n_{c} \times(m-1) n & \left(I_{m-1} \otimes A_{c}\right)
\end{array}\right], \\
& \tilde{B}=\left[\begin{array}{cc}
\bar{L} \otimes\left(B D_{c} K C\right) & 0_{(m-1) n \times(m-1) n_{c}} \\
\bar{L} \otimes\left(B_{c} K C\right) & 0_{(m-1) n_{c} \times(m-1) n_{c}}
\end{array}\right], \\
& \bar{L}=U\left(\sum_{k=1}^{m} L_{k}\right) W \in \mathbb{R}^{(m-1) \times(m-1)} .
\end{aligned}
$$

Hipótese 3. A matriz $\bar{L}=U L W$ possui $m-1$ autovalores reais e é diagonalizável, i.e., existe matriz não singular $Q$ tal que

$$
Q \bar{L} Q^{-1}=\operatorname{diag}\left\{0, \lambda_{2}, \ldots, \lambda_{m-1}\right\} .
$$

A Hipótese 3 restringe o problema à utilização de uma classe específica de grafos direcionados. Li et al. (2018) ressalta que em alguns casos é possível denotar uma topologia que oferece uma matriz laplaciana não diagonalizável por uma matriz laplaciana diagonalizável alterando os pesos do grafo. O mesmo pode acontecer com $\bar{L}$, ao escolher pesos para o grafo, de tal forma que $\bar{L}$ seja diagonalizável.

O Lema de Finsler, apresentado a seguir, será uma ferramenta de grande importância para obter a solução do problema de consenso.

Lema 4. (Skelton et al., 1997) Seja $w(t) \in \mathbb{R}^{n \times n}, \mathcal{Q} \in$ $\mathbb{P}^{n \times n}$ e $\mathbf{B} \in \mathbb{R}^{m \times n}$ tal que $\operatorname{rank}(\mathbf{B})<n$, então as seguintes afirmações são válidas: 
(1) $w(t)^{T} \mathcal{Q} w(t)<0, \mathbf{B} w(t)=0, \forall w(t) \neq 0$.

(2) $\mathbf{B}^{\perp T} \mathcal{Q B}{ }^{\perp}<0$.

(3) $\exists \mu \in \mathbb{R}: \mathcal{Q}-\mu \mathbf{B}^{T} \mathbf{B}<0$.

(4) $\exists \mathcal{X} \in \mathbb{R}^{n \times m}: \mathcal{Q}+\mathcal{X} \mathbf{B}+\mathbf{B}^{T} \mathcal{X}^{T}<0$.

\section{RESULTADOS PRINCIPAIS}

Nesta seção, será apresentada a solução para o problema de consenso de sistemas multiagentes por RDS.

Teorema 5. Considere o sistema multiagente (2), com o protocolo de consenso dinâmico de saída (3)-(4) em uma topologia fixa satisfazendo as Hipóteses 1 e 3. Seja uma dada matriz $K \in \mathbb{R}^{n_{\nu} \times q}$ tal que, $K C$ seja posto linha pleno. Então, o sistema (3)-(4) atinge consenso se existirem as matrizes $P=P^{T}=\left[\begin{array}{cc}P_{11} & 0 \\ 0 & P_{22}\end{array}\right]>0 \in \mathbb{R}^{\left(n+n_{c}\right) \times\left(n+n_{c}\right)}$ e $Z=\left[\begin{array}{ll}Z_{11} & 0\end{array}\right] \in \mathbb{R}^{\left(n_{c}+s\right) \times\left(n+n_{c}\right)}$, que satisfazem a seguinte LMI, para $i=1, \ldots, m-1$ :

$$
\overline{\mathcal{A}}_{i} P+P \overline{\mathcal{A}}_{i}^{T}+\overline{\mathcal{B}}_{i} Z+Z^{T} \overline{\mathcal{B}}_{i}^{T}<0,
$$

onde $\overline{\mathcal{A}}_{i}=\mathcal{V}_{i}^{-1} \mathcal{A} \mathcal{V}_{i}$ e $\overline{\mathcal{B}}_{i}=\mathcal{V}_{i}^{-1} \mathcal{B}$, sendo, $\mathcal{V}_{i}=$ $\left[\mathcal{C}_{i}^{T}\left(\mathcal{C}_{i} \mathcal{C}_{i}^{T}\right)^{-1} \mathcal{C}_{i}^{\perp}\right], \mathcal{C}_{i} \mathcal{V}_{i}=\left[I_{n_{\nu}+n_{c}} 0\right], \mathcal{A}=\left[\begin{array}{cc}A & 0 \\ 0 & 0\end{array}\right], \mathcal{B}=$ $\left[\begin{array}{cc}0 & B \\ I_{n_{c}} & 0\end{array}\right], \mathcal{C}_{i}=\left[\begin{array}{cc}0 & I_{n_{c}} \\ -\lambda_{i} K C & 0\end{array}\right]$.

Se as LMIs (24) forem factíveis, os ganhos dos controladores dinâmicos são obtidos por $\mathcal{K}=Z_{11} P_{11}^{-1}$.

Prova. Definindo $\psi(t)$, tal que, $\psi_{i}(t)=\left[\begin{array}{l}z_{1, i}(t) \\ z_{2, i}(t)\end{array}\right], i=$ $1, \ldots, m-1$, tem-se que $(22)$ pode ser reescrito como

$$
\dot{\psi}(t)=\left(I_{m-1} \otimes\left[\begin{array}{cc}
A & B C_{c} \\
0 & A_{c}
\end{array}\right]-\bar{L} \otimes\left[\begin{array}{cc}
\left(B D_{c} K C\right) & 0 \\
\left(B_{c} K C\right) & 0
\end{array}\right]\right) \psi(t) .
$$

Agora, considere que existe uma matriz não singular $Q$, tal que $Q \bar{L} Q^{-1}=\Lambda$, é uma matriz diagonal. Por meio da transformação $\left(Q \otimes I_{n+n_{c}}\right) \psi(t)=\phi(t)$, tem-se que $(25)$ pode ser reescrito como

$$
\dot{\phi}(t)=\left(I_{m-1} \otimes\left[\begin{array}{cc}
A & B C_{c} \\
0 & A_{c}
\end{array}\right]-\Lambda \otimes\left[\begin{array}{cc}
\left(B D_{c} K C\right) & 0 \\
\left(B_{c} K C\right) & 0
\end{array}\right]\right) \phi(t) .
$$

Uma forma equivalente de $(26)$, para $i=1, \ldots, m-1$, é dada por

$$
\dot{\phi}_{i}(t)=\left[\begin{array}{cc}
A-\lambda_{i} B D_{c} K C & B C_{c} \\
-\lambda_{i} B_{c} K C & A_{c}
\end{array}\right] \phi_{i}(t),
$$

onde, $\lambda_{i}$ são os autovalores de $\bar{L}$.

Observe que (27), pode ser reescrito como um problema de Realimentação Estática de Saída (RES). Então, para $i=1, \ldots, m-1$, tem-se

$$
\dot{\phi}_{i}(t)=(\underbrace{\left[\begin{array}{ll}
A & 0 \\
0 & 0
\end{array}\right]}_{\mathcal{A}}+\underbrace{\left[\begin{array}{cc}
0 & B \\
I_{n_{c}} & 0
\end{array}\right]}_{\mathcal{B}} \underbrace{\left[\begin{array}{ll}
A_{c} & B_{c} \\
C_{c} & D_{c}
\end{array}\right]}_{\mathcal{K}} \underbrace{\left[\begin{array}{cc}
0 & I_{n_{c}} \\
-\lambda_{i} K C & 0
\end{array}\right]}_{\mathcal{C}_{i}}) \phi_{i}(t) .
$$

Assim como proposto em De Oliveira et al. (2002), tomando $\mathcal{V}_{i}=\left[\mathcal{C}_{i}^{T}\left(\mathcal{C}_{i} \mathcal{C}_{i}^{T}\right)^{-1} \mathcal{C}_{i}^{\perp}\right]$, tal que $\mathcal{C}_{i} \mathcal{V}_{i}=\left[I_{n_{\nu}+n_{c}} 0\right]$, então o sistema com variáveis $\mathcal{V}_{i}^{-1} \phi_{i}(t)=\varphi_{i}(t)$ é dado por:

$$
\dot{\varphi}_{i}(t)=\left(\overline{\mathcal{A}}_{i}+\overline{\mathcal{B}}_{i} \mathcal{K} \mathcal{C}_{i} \mathcal{V}_{i}\right) \varphi_{i}(t), i=1, \ldots, m-1,
$$

sendo $\overline{\mathcal{A}}_{i}=\mathcal{V}_{i}^{-1} \mathcal{A} \mathcal{V}_{i}, \overline{\mathcal{B}}_{i}=\mathcal{V}_{i}^{-1} \mathcal{B}$. Para testar a estabilidade do Sistema (29) será considerada a sua forma dual denotada pelo sobrescrito $(.)^{\prime}$. Assim, definida a função candidata de Lyapunov como $V\left(\varphi_{i}^{\prime}(t)\right)=\varphi_{i}^{\prime}(t)^{T} P \varphi_{i}^{\prime}(t)>$ 0, tal que $P=\left[\begin{array}{cc}P_{11} & 0 \\ 0 & P_{22}\end{array}\right]>0$, então

$$
\dot{V}\left(\varphi_{i}^{\prime}(t)\right)=\dot{\varphi}_{i}^{\prime}(t)^{T} P \varphi_{i}^{\prime}(t)+\varphi_{i}^{\prime}(t)^{T} P \dot{\varphi}_{i}^{\prime}(t)<0 .
$$

A partir da desigualdade (30), podemos obter as LMIs (24), definindo $Z=[\mathcal{K} 0] P$ e por conseguinte, $Z_{11}=$ $\mathcal{K} P_{11}$. Caso haja factibilidade na solução do problema, os ganhos $A_{c}, B_{c}, C_{c}$ e $D_{c}$ dos controladores dinâmicos (3) são obtidos através de $\mathcal{K}=Z_{11} P_{11}^{-1}$. Os ganhos obtidos estabilizam o sistema (29), o que garante consenso ao sistema (2).

Observação 6. As condições oferecidas são LMIs mediante uma escolha prévia de $K$. Caso $C$ seja posto linha pleno, é possível escolher $K=I$ sem perda de generalidade. Quando $C$ não é posto linha pleno, devemos escolher $K$ com posto linha pleno, levando em consideração que $\operatorname{rank}(K C) \leq \min \{\operatorname{rank}(K), \operatorname{rank}(C)\}$ (Meyer, 2000). Como $K$ é escolhida, sempre teremos uma $K$ que garante posto linha pleno para $K C$.

Condições LMI menos conservadoras para o projeto de (3) podem ser obtidas pela introdução de variáveis de folga, por meio do Lema 4, evitando restrições na estrutura da matriz de Lyapunov, conforme o teorema a seguir.

Teorema \%. Considere o sistema multiagente (2), com o protocolo de consenso dinâmico de saída (3)-(4) em uma topologia fixa satisfazendo as Hipóteses 1 e 3. Seja uma dada matriz $K \in \mathbb{R}^{n_{\nu} \times q}$ tal que, $K C$ seja posto linha pleno. Então, o sistema (3)-(4) atinge consenso se para algum escalar $\xi>0$, existem matrizes $P=P^{T}>0$, $X=\left[\begin{array}{cc}X_{11} & 0 \\ X_{21} & X_{22}\end{array}\right] \in \mathbb{R}^{\left(n+n_{c}\right) \times\left(n+n_{c}\right)}, Z=\left[\begin{array}{ll}Z_{11} & 0\end{array}\right] \in$ $\mathbb{R}^{\left(n_{c}+s\right) \times\left(n+n_{c}\right)}$, que satisfazem as seguintes LMIs para $i=1, \ldots, m-1$ :

$$
\left[\begin{array}{cc}
\overline{\mathcal{A}}_{i} X+X^{T} \overline{\mathcal{A}}_{i}^{T}+\overline{\mathcal{B}}_{i} Z+Z^{T} \overline{\mathcal{B}}_{i}^{T} & \star \\
\left(P-X^{T}+\xi \overline{\mathcal{A}}_{i} X+\xi \overline{\mathcal{B}}_{i} Z\right)^{T} & -\xi X^{T}-\xi X
\end{array}\right]<0,
$$

onde $\overline{\mathcal{A}}_{i}=\mathcal{V}_{i}^{-1} \mathcal{A} \mathcal{V}_{i}$ e $\overline{\mathcal{B}}_{i}=\mathcal{V}_{i}^{-1} \mathcal{B}$, sendo, $\mathcal{V}_{i}=$ $\left[\mathcal{C}_{i}^{T}\left(\mathcal{C}_{i} \mathcal{C}_{i}^{T}\right)^{-1} \mathcal{C}_{i}^{\perp}\right], \mathcal{C}_{i} \mathcal{V}_{i}=\left[I_{n_{\nu}+n_{c}} 0\right], \mathcal{A}=\left[\begin{array}{cc}A & 0 \\ 0 & 0\end{array}\right], \mathcal{B}=$ $\left[\begin{array}{cc}0 & B \\ I_{n_{c}} & 0\end{array}\right], \mathcal{C}_{i}=\left[\begin{array}{cc}0 & I_{n_{c}} \\ -\lambda_{i} K C & 0\end{array}\right]$

Se as LMIs (31) forem factíveis, os ganhos dos controladores dinâmicos são obtidos por $\mathcal{K}=Z_{11} X_{11}^{-1}$.

Prova. Observe que a desigualdade (30) pode ser reescrita da seguinte forma

$$
\underbrace{\left[\begin{array}{c}
\varphi_{i}^{\prime}(t) \\
\dot{\varphi}_{i}^{\prime}(t)
\end{array}\right]^{T}}_{w(t)^{T}} \underbrace{\left[\begin{array}{cc}
0 & P \\
P & 0
\end{array}\right]}_{\mathcal{Q}} \underbrace{\left[\begin{array}{c}
\varphi_{i}^{\prime}(t) \\
\dot{\varphi}_{i}^{\prime}(t)
\end{array}\right]}_{w(t)}<0
$$

tal que,

$$
\underbrace{\left[\left(\overline{\mathcal{A}}_{i}+\overline{\mathcal{B}}_{i} \mathcal{K} \mathcal{C}_{i} \mathcal{V}_{i}\right)^{T}-I\right]}_{\mathbf{B}} \underbrace{\left[\begin{array}{c}
\varphi_{i}^{\prime}(t) \\
\dot{\varphi}_{i}^{\prime}(t)
\end{array}\right]}_{w(t)}=0 .
$$


A partir das identificações anteriores e do item (4) do Lema 4, é obtida a seguinte desigualdade

$$
\left[\begin{array}{ll}
0 & P \\
P & 0
\end{array}\right]+H e\left\{\left[\begin{array}{l}
X_{1} \\
X_{2}
\end{array}\right]\left[\left(\overline{\mathcal{A}}_{i}+\overline{\mathcal{B}}_{i} \mathcal{K C}_{i} \mathcal{V}_{i}\right)^{T}-I\right]\right\}<0
$$

sendo $X_{1}, X_{2} \in \mathbb{R}^{\left(n+n_{c}\right) \times\left(n+n_{c}\right)}$. Portanto, segue que,

$$
\left[\begin{array}{cc}
\Psi_{i} X_{1}^{T}+X_{1} \Psi_{i}^{T} & \star \\
P-X_{1}^{T}+X_{2} \Psi_{i}^{T}-X_{2}-X_{2}^{T}
\end{array}\right]<0
$$

onde, $\Psi_{i}=\overline{\mathcal{A}}_{i}+\overline{\mathcal{B}}_{i} \mathcal{K} \mathcal{C}_{i} \mathcal{V}_{i}$.

Para recuperar $\mathcal{K}$ é necessário definir $X_{1}=X^{T}$ e $X_{2}=$ $\xi X^{T}$, para $\xi>0$. Assim, é obtida a seguinte LMI

$$
\left[\begin{array}{cc}
\overline{\mathcal{A}}_{i} X+X^{T} \overline{\mathcal{A}}_{i}^{T}+\overline{\mathcal{B}}_{i} Z+Z^{T} \overline{\mathcal{B}}_{i}^{T} & \star \\
P^{T}-X+\xi X^{T} \overline{\mathcal{A}}_{i}^{T}+\xi Z^{T} \overline{\mathcal{B}}_{i}^{T} & -\xi X^{T}-\xi X
\end{array}\right]<0,
$$

onde $Z=\mathcal{K C}_{i} \mathcal{V}_{i} X$ é uma variável definida com o intuito de linearizar a desigualdade (35). Impondo uma estrutura particular à variável $X$ e considerando $Z=\left[\begin{array}{ll}Z_{11} & 0\end{array}\right]$, tem-se que:

$$
\begin{aligned}
{\left[\begin{array}{ll}
Z_{11} & 0
\end{array}\right] } & =\mathcal{K}\left[\begin{array}{ll}
I_{n_{\nu}+n_{c}} & 0
\end{array}\right]\left[\begin{array}{cc}
X_{11} & 0 \\
X_{21} & X_{22}
\end{array}\right] \\
& =\left[\begin{array}{ll}
\mathcal{K} X_{11} & 0
\end{array}\right]
\end{aligned}
$$

Da igualdade anterior, segue que $\mathcal{K} X_{11}=Z_{11}$ e, portanto, $\mathcal{K}=Z_{11} X_{11}^{-1}$.

Observou-se numericamente que o Teorema 7 fornece, para alguns casos, valores nulos para $B_{c}$ e $C_{c}$ recuperando um controlador estático de saída, ao invés de dinâmico, no projeto de (3)-(4) (Morais et al., 2016). Uma estratégia para contornar essa dificuldade na obtenção de ganhos $B_{c}$ e $C_{c}$ não nulos, é introduzir uma matriz $\Gamma$ não singular, tal que $\overline{\mathcal{A}}_{i}+\overline{\mathcal{B}}_{i} \mathcal{K} \mathcal{C}_{i} \mathcal{V}_{i}=\overline{\mathcal{A}}_{i}+\overline{\mathcal{B}}_{i} \Gamma^{-1} \Gamma \mathcal{K} \mathcal{C}_{i} \mathcal{V}_{i}$, como será apresentado no próximo corolário.

Corolário 8. Considere o sistema multiagente (2), com o protocolo de consenso dinâmico de saída (3)-(4) em uma topologia fixa satisfazendo as Hipóteses 1 e 3. Seja uma dada matriz $K \in \mathbb{R}^{n_{\nu} \times q}$ tal que, $K C$ seja posto linha pleno. Então, o sistema (3)-(4) atinge consenso se para os escalares $\xi>0$ e $\beta \neq 0$, existem matrizes $P=P^{T}>0$, $X=\left[\begin{array}{cc}X_{11} & 0 \\ X_{21} & X_{22}\end{array}\right] \in \mathbb{R}^{\left(n+n_{c}\right) \times\left(n+n_{c}\right)}, M_{1} \in \mathbb{R}^{n_{c} \times n_{c}}, M_{2} \in$ $\mathbb{R}^{s \times\left(n-n_{c}\right)}$ e $M_{3}, Q \in \mathbb{R}^{s \times n_{c}}$ que satisfazem as seguintes LMIs para $i=1, \ldots, m-1$ :

$$
\left[\begin{array}{cc}
\overline{\mathcal{A}}_{i} X+X^{T} \overline{\mathcal{A}}_{i}^{T}+\overline{\mathcal{B}}_{i} Z+Z^{T} \overline{\mathcal{B}}_{i}^{T} & \star \\
\left(P-X^{T}+\xi \overline{\mathcal{A}}_{i} X+\xi \overline{\mathcal{B}}_{i} Z\right)^{T} & -\xi X^{T}-\xi X
\end{array}\right]<0,
$$

onde $Z=\left[\begin{array}{ll}Z_{11} & 0\end{array}\right], Z_{11}=\left[\begin{array}{cc}\beta M_{1} R & M_{1} \\ \beta Q R+M_{2} & Q+M_{3}\end{array}\right], R=$ $\left[I_{n_{c}} 0_{n_{c} \times\left(n-2 n_{c}\right)}\right], \overline{\mathcal{A}}_{i}=\mathcal{V}_{i}^{-1} \mathcal{A} \mathcal{V}_{i}, \overline{\mathcal{B}}_{i}=\mathcal{V}_{i}^{-1} \mathcal{B}, \mathcal{V}_{i}=$ $\left[\mathcal{C}_{i}^{T}\left(\mathcal{C}_{i} \mathcal{C}_{i}^{T}\right)^{-1} \mathcal{C}_{i}^{\perp}\right], \mathcal{C}_{i} \mathcal{V}_{i}=\left[\begin{array}{ll}I_{n_{\nu}+n_{c}} & 0\end{array}\right]$, sendo, $\mathcal{A}=\left[\begin{array}{cc}A & 0 \\ 0 & 0\end{array}\right]$, $\mathcal{B}=\left[\begin{array}{cc}0 & B \\ I_{n_{c}} & 0\end{array}\right], \mathcal{C}_{i}=\left[\begin{array}{cc}0 & I_{n_{c}} \\ -\lambda_{i} K C & 0\end{array}\right]$.

Se as LMIs anteriores forem factíveis, os ganhos dos controladores dinâmicos são obtidos por $\mathcal{K}=Z_{11} X_{11}^{-1}$.

Prova. Observe que:

$$
\begin{aligned}
& \overline{\mathcal{A}}_{i} X+\overline{\mathcal{B}}_{i} \mathcal{K} \mathcal{C}_{i} \mathcal{V}_{i} X=\overline{\mathcal{A}}_{i} X+\overline{\mathcal{B}}_{i} \Gamma^{-1} \Gamma \mathcal{K} \mathcal{C}_{i} \mathcal{V}_{i} X \\
& =\overline{\mathcal{A}}_{i} X+\overline{\mathcal{B}}_{i}\left[\Gamma^{-1} \Gamma \mathcal{K} X_{11} 0\right],
\end{aligned}
$$

sendo $M=\Gamma \mathcal{K} X_{11}$ tal que $M=\left[\begin{array}{cc}\beta M_{1} R & M_{1} \\ M_{2} & M_{3}\end{array}\right]$ e $\Gamma=$ $\left[\begin{array}{cc}I & 0 \\ -Y & I\end{array}\right]$. Daí, segue que

$$
\mathcal{K} X_{11}=\Gamma^{-1} M=\left[\begin{array}{cc}
\beta M_{1} R & M_{1} \\
\beta Q R+M_{2} & Q+M_{3}
\end{array}\right], Q=Y M_{1} .
$$

Definindo $Z_{11}=\mathcal{K} X_{11}$, então $\mathcal{K}=Z_{11} X_{11}^{-1}$.

Neste trabalho, a principal contribuição é fornecer condições convexas para o projeto de protocolo de consenso por um controlador dinâmico de saída e a incorporação de um ganho $K$ no protocolo de consenso, onde $K C$ é considerado posto linha pleno. Dessa forma, evita-se restrições de posto nas matrizes $B$ e $C$ dos agentes em (2).

\section{EXEMPLO NUMÉRICO}

Com o intuito de mostrar a aplicação das condições propostas em alcançar consenso em problemas práticos, será apresentado um exemplo numérico nessa seção.

Para a simulação será considerado um sistema robótico de segunda ordem, que representa a dinâmica de quadrotores, apresentado em Schwager et al. (2011), descrito pela seguinte equação linear no espaços de estados:

$$
\begin{gathered}
{\left[\begin{array}{l}
\dot{p}_{i}(t) \\
\ddot{p}_{i}(t)
\end{array}\right]=\underbrace{\left[\begin{array}{cc}
0 & 1 \\
-c & -b
\end{array}\right]}_{A}\left[\begin{array}{l}
p_{i}(t) \\
\dot{p}_{i}(t)
\end{array}\right]+\underbrace{\left[\begin{array}{ll}
0 & 0 \\
1 & 2
\end{array}\right]}_{B} u_{i}(t),} \\
y_{i}(t)=\underbrace{\left[\begin{array}{cc}
1 & 0 \\
2 & 0
\end{array}\right]}_{C}\left[\begin{array}{l}
p_{i}(t) \\
\dot{p}_{i}(t)
\end{array}\right], i=1, \ldots, 6 .
\end{gathered}
$$

Observe que $B$ não possui posto coluna completo e $C$, da mesma forma, não possui posto linha completo. Serão considerados $\mathrm{O}$ ganho do protocolo de consenso (4) foi definido como $K=\left[\begin{array}{ll}1 & 2\end{array}\right]$, de maneira que $K C$ é posto linha pleno, além disso, $c=0$ e $b=1.5$.

Serão considerados 6 quadrotores, distribuídos em uma rede de comunicação modelada pelo grafo direcionado mostrado na Figura 1.

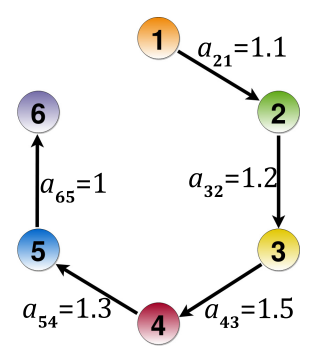

Figura 1. Grafo direcionado que modela a comunicação.

O Corolário 8 foi solucionado com o pacote SeDuMi, utilizando o toolbox YALMIP, no software Matlab R2016a, em um computador com sistema operacional Windows 10 . Para $\xi=0.5$ e $\beta=0.5$, foram encontrados os seguintes ganhos para os controladores dinâmicos

$$
\begin{aligned}
& A_{c}=-0.6520, B_{c}=-0.3541 \\
& C_{c}=\left[\begin{array}{c}
0.0478 \\
0
\end{array}\right], D_{c}=\left[\begin{array}{c}
0.3754 \\
0
\end{array}\right] .
\end{aligned}
$$


A resposta temporal dos agentes com condições iniciais mostradas na Tabela 1 para o protocolo projetado é mostrado na Figura 2. Conforme pode ser observado, os agentes alcançam consenso.

Com a mesma metodologia e considerando $\xi=0.5$, o Teorema 7 encontrou solução fornecendo, contudo, valores nulos para $B_{c}$ e $C_{c}$, e, portanto, retornando um controlador estático ao invés de um controlador dinâmico de saída. O Teorema 5 não apresentou solução para (39)-(40), mostrando que o Teorema 7 e o Corolário 8 são menos conservadores.
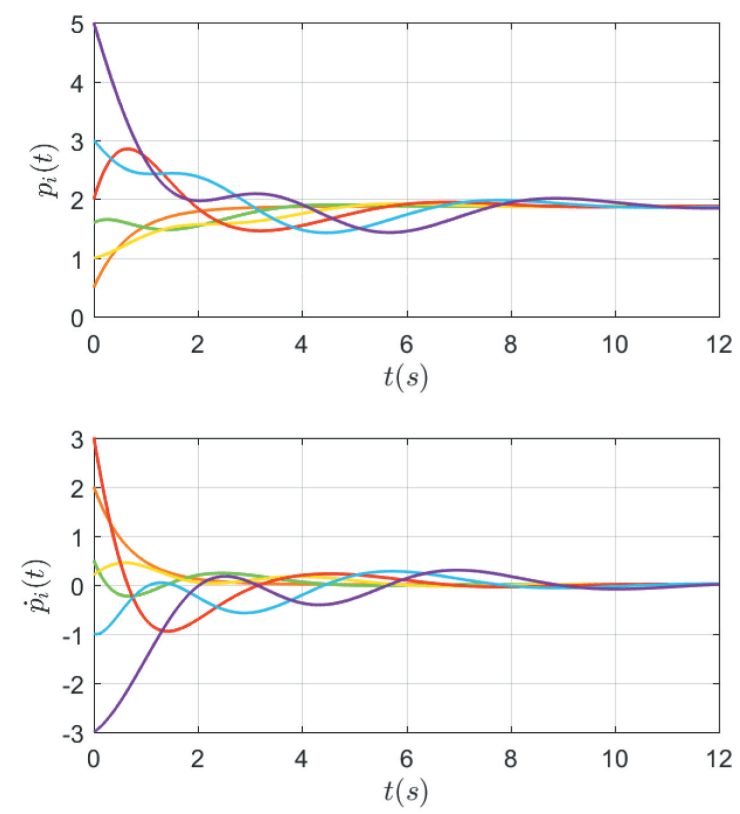

Figura 2. Consenso dos agentes em relação à posição $p_{i}(t)$ e velocidade $\dot{p}_{i}(t)$ em um grau de liberdade, considerando o controlador dinâmico (3) e os ganhos dados em (41).

Tabela 1. Condições iniciais de cada agente.

\begin{tabular}{ccccccc} 
Agente & 1 & 2 & 3 & 4 & 5 & 6 \\
\hline$p_{i}(0)$ & 0.5 & 1.6 & 1 & 2 & 3 & 5 \\
$\dot{p}_{i}(0)$ & 2 & 0.5 & 0.2 & 3 & -1 & -3 \\
\hline
\end{tabular}

\section{CONCLUSÕES}

Neste artigo foram propostas novas condições suficientes para o problema de consenso de sistemas multiagentes quando a informação dos estados não está disponível para troca entre os agentes. O método proposto torna possível o projeto de um protocolo de consenso com realimentação dinâmica de saída para agentes dinâmicos lineares e homogêneos. Diferentemente da literatura, as condições de projeto não exigem restrição de posto nas matrizes de entrada ou de saída dos agentes. É esperado que este resultado, possa ser estendido para casos mais complexos encontrados na literatura como por exemplo sistemas multi-agentes sujeitos a atrasos de comunicação e mudança de topologia da rede que conecta os agentes.

\section{REFERÊNCIAS}

Ao, D., Yang, G., and Wang, X. (2018). $H_{\infty}$ Consensus Control of Multi-Agent Systems: A Dynamic Output
Feedback Controller with Time-Delays. In 2018 Chinese Control And Decision Conference (CCDC), 4591-4596. IEEE.

De Oliveira, M.C., Geromel, J.C., and Bernussou, J. (2002). Extended $H_{2}$ and $H_{\infty}$ norm characterizations and controller parametrizations for discrete-time systems. International Journal of Control, 75(9), 666-679.

Dorri, A., Kanhere, S.S., and Jurdak, R. (2018). Multiagent systems: A survey. IEEE Access, 6, 28573-28593.

Gao, L., Tong, C., and Wang, L. (2014). $H_{\infty}$ Dynamic Output Feedback Consensus Control for Discrete-Time Multi-Agent Systems with Switching Topology. Arabian Journal for Science and Engineering, 39(2), 1477-1487.

Godsil, C. and Royle, G.F. (2001). Algebraic graph theory, volume 207. Springer Science \& Business Media.

Kolaric, P., Chen, C., Dalal, A., and Lewis, F.L. (2018). Consensus controller for multi-uav navigation. Control Theory and Technology, 16(2), 110-121.

Li, X., Soh, Y.C., and Xie, L. (2018). Robust consensus of uncertain linear multi-agent systems via dynamic output feedback. Automatica, 98, 114-123.

Liu, Y. and Jia, Y. (2010). $H_{\infty}$ Consensus control of multiagent systems with switching topology: A dynamic output feedback protocol. International Journal of Control, $83(3), 527-537$.

Meyer, C.D. (2000). Matrix analysis and applied linear algebra, volume 71. Siam.

Min, Y., Luan, X., Ding, Z., and Liu, F. (2018). Giventime consensus for stochastic Markov jump networks by dynamic output feedback. Transactions of the Institute of Measurement and Control, 40(10), 3160-3168.

Morais, C.F., Braga, M.F., Oliveira, R.C., and Peres, P.L. (2016). LMI-based design of $H_{\infty}$ dynamic output feedback controllers for MJLS with uncertain transition probabilities. In 2016 American Control Conference (ACC), 5650-5655. IEEE.

Olfati-Saber, R., Fax, J.A., and Murray, R.M. (2007). Consensus and cooperation in networked multi-agent systems. Proceedings of the IEEE, 95(1), 215-233.

Ren, C.E., Du, T., Li, G., and Shi, Z. (2018). Disturbance observer-based consensus control for multiple robotic manipulators. IEEE Access, 6, 51348-51354.

Richter, S. and DeCarlo, R. (1984). A homotopy method for eigenvalue assignment using decentralized state feedback. IEEE transactions on automatic control, 29(2), 148-158.

Schwager, M., Michael, N., Kumar, V., and Rus, D. (2011). Time scales and stability in networked multirobot systems. In 2011 IEEE International Conference on Robotics and Automation, 3855-3862. IEEE.

Skelton, R.E., Iwasaki, T., and Grigoriadis, D.E. (1997). A unified algebraic approach to control design. CRC Press.

Sun, Y.G. and Wang, L. (2009). Consensus of multiagent systems in directed networks with nonuniform time-varying delays. IEEE Transactions on Automatic Control, 54(7), 1607-1613.

Zhang, H. and Gurfil, P. (2018). Cooperative orbital control of multiple satellites via consensus. IEEE Transactions on Aerospace and Electronic Systems, 54(5), 2171-2188.

Zhao, H. and Park, J.H. (2015). Dynamic output feedback consensus of continuous-time networked multiagent systems. Complexity, 20(5), 35-42. 